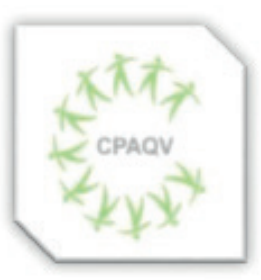

ISSN: 2178-7514

Vol. $12\left|\mathbf{N}^{\circ} .3\right|$ Ano 2020
ARTIGO ORIGINAL

\section{ANÁLISE COMPARATIVA DOS EFEITOS DA FISIOTERAPIA AQUÁTICA E DA MUSCULAÇÃO TERAPEUTICA NA QUALIDADE DE VIDA E NO EQUILÍBRIO DE IDOSOS}

\author{
Comparative analysis of the effects of aquatic physiotherapy and \\ therapeutic musculation in the quality of life and balance of elderly
}

Jéssica Patrícia da Silva Alves ${ }^{1}$; Prisciane Anastacia da Silva Nascimento ${ }^{2}$; Aymee Lobato Brito ${ }^{3}$; Tainá Alves Teixeira ${ }^{4}$; Marcio Clementino de Souza Santos ${ }^{5}$; Luciane Lobato Sobral ${ }^{6}$

\begin{abstract}
RESUMO
Introdução: Envelhecer com qualidade de vida é um grande desafio e, com isso, vem a preocupação dos profissionais da saúde em minimizar os efeitos negativos do envelhecimento, os quais devem orientar o paciente sobre a importância de conhecer as maneiras de obter o controle e a manutenção da funcionalidade. Devido ao grande número de fatores que afetam o equilíbrio, a prática de exercícios físicos está sendo sugerida como forte indicador de melhora de qualidade de vida. Objetivo: Analisar os resultados gerados da comparação entre os efeitos da fisioterapia aquática e da musculação terapêutica no equilibrio e na qualidade de vida de idosos. Metodologia: Foram selecionados 40 pacientes, divididos em 4 grupos, sendo distribuídos da seguinte forma: Grupo FA (fisioterapia aquática); Grupo MT (musculação terapêutica); Grupo MT+FA (fisioterapia aquática mais musculação terapêutica) e Grupo controle, classificados como idosos sedentários da comunidade. Foi coletada a assinatura do TCLE e aplicada fichas de avaliações e escalas de equilíbrio (Tinetti), qualidade de vida (Sf-36), eficácia de quedas -Internacional (FES I). Resultados: Os recursos fisioterapêuticos utilizados em ambiente aquático e terrestre apresentam uma significante melhora na manutenção do equilíbrio. Entretanto, estudos sobre esse tema ainda são bastante escassos, assim como o termo "musculação terapêutica", o qual ainda é pouco conhecido e mencionado na literatura. Em virtude disso, o presente estudo tem o intuito de contribuir com mais informações sobre este tema na tentativa de preencher essa lacuna de conhecimento. Conclusão: A atividade física independentemente do tipo promove efeitos benéficos na qualidade de vida.
\end{abstract}

Palavras-chave: Idoso, fisioterapia, hidroterapia, musculação terapêutica, qualidade de vida.

\begin{abstract}
Introduction: Aging with quality of life is a major challenge, and with this, come concern of the health professionals in reduce the negative effects of aging that have guide the patients about conyrol and maintenance of functionatility. Due to the great number of factors that affect balance, the practice of physical exercises is being suggested as a strong indicator of improvement in quality of life. Objective: To analyze the results generated from the comparison of the effects of aquatic physiotherapy and bodybuilding therapy on the balance and quality of life of the elderly. Methodology: 40 patients were selected, divided into 4 groups, being distributed as follows: Group FA (aquatic physiotherapy); MT group (therapeutic weight training); Group MT + FA (aquatic physiotherapy plus therapeutic bodybuilding) and control group, classified as sedentary elders of the community. The TCLE was collected and evaluation sheets and balance scales were applied: Tinetti, quality of life (Sf-36), efficacy of fall -International (FES I). Results: The physiotherapeutic resources used in the aquatic environment and used in terrestrial environment presents a significant improvement and maintenance of the balance. However, studies on this subject are still scarce, as is the term "bodybuilding therapy", which is still little known and mentioned in the literature. Because of this, the present study was designed with the intention of contributing more information about this attempt to fill this knowledge gap. Conclusion: The physical activity independent of type promotes beneficial effects on quality of life.
\end{abstract}

Keywords: Elderly, physiotherapy, hydrotherapy, therapeutic bodybuilding, quality of life

\footnotetext{
1 Fisioterapeuta graduada pela Universidade da Amazônia (UNAMA). Belém-PA. Email: jessicapatricia-tga@hotmail.com

2 Fisioterapeuta graduada pela Universidade da Amazônia (UNAMA). Belém-PA. Email: priscianen@gmail.com

3 Fisioterapeuta graduada pela Universidade da Amazônia (UNAMA). Belém-PA. Email: brito.aymee20@gmail.com.

4 Docente pela Universidade Estadual do Pará - UEPA. Belém-PA. Email: tainaleonardo@hotmail.com

5 Doutor em Doenças Tropicais pela Universidade Federal do Pará - UFPA. Email: marcio.clementino@gmail.com.

6 Doutora em Ciências do Movimento Humano pela Unimep- SP. Email: lucianelobatosobral@gmail.com
}

Autor de correspondência

Aymee Lobato Brito

brito.aymee20@gmail.com

DOI: $\underline{10.36692 / v 12 n 3-41}$ 


\section{INTRODUÇÃO}

Atualmente, envelhecer com qualidade de vida é um grande desafio ${ }^{1,2,3}$, com isso, vem a preocupação dos profissionais da saúde em minimizar os efeitos do envelhecimento. Para esses profissionais, não cabe somente a tarefa de propor, mas de sensibilizar por meio de políticas de prevenções a orientar o paciente sobre a importância de conhecer as maneiras de obter o controle e a manutenção da funcionalidade, ${ }^{4,5}$ retardar e diminuir o processo de incapacidade funcional que possa dificultar ou impedir o desempenho de atividades de vida diária. ${ }^{6}$

Devido ao grande número de fatores que afetam o equilíbrio, a prática de exercícios físicos está sendo sugerida como forte indicador de melhora de qualidade de vida.7,8Através da fisioterapia é possível aplicar programas de treinamento de força e equilíbrio ${ }^{9,10} \mathrm{em}$ ambiente terrestre e aquático, ${ }^{11}$ que podem ser adquiridas e/ ou mantidas por meio da fisioterapia aquática ${ }^{12}$ e do treino resistido, através da musculação terapêutica. ${ }^{13}$

A Fisioterapia aquática permite criar momentos de instabilidade com utilização dos efeitos da turbulência, de modo a suprir grande quantidade de informações sensoriais, promovendo melhora nas reações de equilíbrio do corpo ${ }^{14,15}$. Além disso, quando voltada para o atendimento da terceira idade, ela objetiva a diminuição das dores existentes, aumento da força muscular e melhora do equilíbrio. ${ }^{16,17}$

O exercício resistido é responsável tanto pela prevenção de perda de massa e força muscular quanto para o ganho ou manutenção da mesma, desempenhando um importante papel na manutenção da capacidade funcional do idoso. ${ }^{18,19}$ Atualmente, para tratar doenças e diversas disfunções musculoesqueléticas, os fisioterapeutas vêm utilizando métodos de treinamento resistindo, denominando-se musculação terapêutica, um tema novo na literatura e escasso ainda de estudos inerentes ao mesmo. ${ }^{20,21,22}$

Dessa forma, o respectivo estudo tem por finalidade analisar os resultados gerados da comparação dos efeitos da fisioterapia aquática e musculação terapêutica no equilíbrio e na qualidade de vida de idosos.

\section{MÉTODOS}

O presente estudo foi realizado na Clínica Corpo e Saúde, na cidade de Belém do Pará. A pesquisa foi realizada somente após o aceite do Comitê de Ética em Pesquisa da Universidade da Amazônia - UNAMA sob o protocolo 2.509.973 e após assinatura do termo de consentimento livre e esclarecido (TCLE) pelos participantes.

O estudo caracterizou-se como uma pesquisa observacional transversal, sendo realizado no mês de maio a junho de 2018 . Foram selecionados 40 pacientes voluntários, divididos em 4 grupos, com 10 pacientes em cada distribuídos da seguinte forma: Grupo Fisioterapia Aquática (FA); Grupo Musculação Terapêutica (MT); Grupo MT+FA, 
representados por praticantes das duas modalidades e Grupo Controle (GC) que não realizavam nenhuma das modalidades, sendo classificados como idosos sedentários.

Os participantes incluídos no estudo foram voluntários com idade superior a 60 e inferior 75 anos, de ambos os sexos, que apresentavam maior índice de independência: marcha independente, Mini Exame do Estado Mental (MEEM) de acordo com a escolaridade do paciente, e no mínimo três meses de prática nas modalidades de fisioterapia aquática ou musculação terapêutica.

Foram excluídos participantes que faziam uso de dispositivos de locomoção, que apresentavam incontinência urinária ou fecal, feridas abertas, doenças cutâneas contagiosas, doenças infecciosas, Hipertensão Arterial (PA) não controlada e dispnéia aos mínimos esforços.

A primeira etapa da pesquisa, após o aceite do CEP, foi a divisão dos grupos e assinatura do Termo de Consentimento Livre e Esclarecido (TCLE). Em seguida, foi realizada a coleta de dados da pesquisa no período de maio a junho de 2018, durante duas vezes por semana no turno da manhã e tarde.

No turno matutino foram coletados os dados da FA, no turno da tarde da MT e nos demais dias da semana foram coletados os dados do GC na comunidade, por meio de fichas de avaliação e escalas, as quais foram: equilíbrio (Tinetti), qualidade de vida (Sf-36) e eficácia de queda -Internacional (FES I).

Após a coleta realizou-se a última etapa, que foi a análise estatística por meio do programa Bioestat 5.0, o qual foi utilizado teste de normalidade de D'agostimo Person, Anova de duas vias seguido de post hoc de Bonfferroni com resultados convertidos e fidedignos.

\section{RESULTADOS}

Os resultados das escalas de Tinetti foram analisados de acordo com a média e o desvio padrão dos escores e estão apresentados na tabela 1.

Tabela 1: Média tdesvio padrão da escala de Tinetti de acordo com os grupos.

\begin{tabular}{llll}
\hline GRUPOS & EQUILÍBRIO & MARCHA & TOTAL \\
\hline CONT & $9,8 \pm 3,88$ & $7,9 \pm 2,23$ & $17,9 \pm 5,4$ \\
MT & $11,7 \pm 2,05$ & $7,3 \pm 0,82$ & $19,0 \pm 1,94$ \\
FA & $10,7 \pm 3,77$ & $9,6 \pm 2,07$ & $20,4 \pm 5,1$ \\
MT + FA & $11,4 \pm 2,83$ & $9,0 \pm 3,39$ & $20,9 \pm 3,28$ \\
\hline
\end{tabular}

Fonte: dados da pesquisa

CONT: controle, MT: musculação terapêutica, FA: Fisioterapia Aquática, MT + FA: Musculação terapêutica e fisioterapia aquática. 
$\mathrm{Na}$ avaliação de equilíbrio, o grupo MT apresentou melhores resultados quanto aos demais grupos $(11,7 \pm 2,05)$. Na marcha, o grupo FA apresentou melhor desempenho $(9,6 \pm 2,07)$. No entanto, na avaliação total (equilíbrio e marcha), o grupo MT + FA obteve uma diferença significativa $(20,9 \pm 3,28)$ em relação aos outros grupos $(\mathrm{P}<0,05)$.

Os resultados da escala do SF-36 foram analisados de acordo com a média e o desvio padrão dos escores e estão apresentados nas tabelas 2 e 3 .

Tabela 2: Média \pm desvio padrão da escala SF-36 de acordo com os grupos

\begin{tabular}{llllll}
\hline GRUPOS & C. F & L.F & DOR & E.G.S & P \\
\hline CONT & $17,5 \pm 10,9$ & $13,4 \pm 17,4$ & $32,3 \pm 13,1$ & $37,6 \pm 19,1$ & $\mathrm{p}<0,05$ \\
MT & $44,0 \pm 16,8$ & $40,1 \pm 7,60$ & $33,8 \pm 23,2$ & $43,7 \pm 40,1$ & $\mathrm{P}<0,05$ \\
FA & $40,5 \pm 14,5$ & $37,4 \pm 31,5$ & $56,8 \pm 19,6$ & $37,8 \pm 18,7$ & $\mathrm{P}<0,05$ \\
MT+FA & $35,5 \pm 21,2$ & $49,9 \pm 36,4$ & $53,9 \pm 28,3$ & $52,7 \pm 20,9$ & $\mathrm{P}<0,05$ \\
\hline
\end{tabular}

Fonte: Dados da pesquisa

CONT: controle, MT: musculação terapêutica, FA: Fisioterapia Aquática, MT + FA: Musculação terapêutica e fisioterapia aquática, C.F: capacidade funcional, L.F: limitação por aspectos físicos, E.G.S: estado geral de saúde.

$\mathrm{Na}$ qualidade de vida, o grupo MT + FA na capacidade funcional e vitalidade, enquanto apresentou melhores resultados para limitações a FA apresentou uma diferença significativa física, estado geral de saúde e aspectos sociais, o em relação a dor, aspectos emocionais e saúde grupo MT apresentou uma diferença significativa mental.

\begin{tabular}{lc}
\multicolumn{2}{l}{ Tabela 3: Média \pm desvio padrão da escala de eficácia de queda. } \\
\hline GRUPOS & Média \pm DP \\
\hline CONT & $35.4 \pm 4,72$ \\
MT & $29.9 \pm 8,69$ \\
FA & $30.9 \pm 10.1$ \\
MT+FA & $24.2 \pm 6.73$ \\
\hline
\end{tabular}

Fonte: Dados da Pesquisa

CONT: controle, MT: musculação terapêutica, FA: Fisioterapia Aquática, MT + FA: Musculação terapêutica e fisioterapia aquática.

O maior índice de medo e de queda deu-se no grupo controle, infere-se esse medo devido ao baixo índice de marcha dinâmica dos idosos, podendo acarretar o comprometimento progressivo da capacidade funcional e favorecer ainda mais o sedentarismo. Houve significância entre os grupos $(\mathrm{P}<0,05)$.

\section{DISCUSSÃO}

Os achados do presente estudo apontaram que o grupo FA apresentou melhores resultados de marcha comparado aos demais grupos. Este resultado sugere que o ganho e manutenção da marcha em ambiente aquático apresentou mais relevância que em ambiente terrestre. Segundo 
o estudo de Resende ${ }^{23}$ o déficit de equilíbrio e desordens na marcha, dentre outras disfunções, dificultam a realização dos exercícios em ambiente terrestre por idosos, ao contrário dos exercícios realizados no meio aquático, onde há diminuição da sobrecarga articular, menor risco de quedas e de lesões.

A flutuação possibilita ao indivíduo realizar exercícios e movimentos que não podem ser realizados no solo. ${ }^{12,14} \mathrm{~A}$ importância da especificidade e do treinamento de força, equilíbrio e redução da dor na fisioterapia aquática foi demonstrada nos achados de Coehn ${ }^{12}$ e Resende. ${ }^{14}$

Os autores André ${ }^{23}$ e Souza ${ }^{24}$ sugerem que idosos praticantes de musculação possuem melhor equilíbrio dinâmico e capacidade de produção de força nos membros inferiores em relação a idosos praticantes de atividade em meio aquático. Esses dados corroboram com a presente pesquisa em que o grupo da musculação terapêutica apresenta valores maiores no item equilíbrio da escala de Tinetti.

Nessa perspectiva, o estudo sugere que os praticantes de MT apresentam melhor capacidade funcional no SF-36. A literatura tem mostrado evidências de que o exercício resistido é eficaz para a manutenção, ganho de força muscular, mobilidade e capacidade funcional de idosos, contudo, estes estudos são conduzidos comparando idosos que praticam treinamento resistido contra indivíduos sedentários. ${ }^{25}$
Sobretudo, o grupo de FA na combinação da MT apresentaram melhor equilíbrio quando comparado com os outros grupos. O estudo sugere que os princípios e recursos fisioterapêuticos utilizados em ambiente aquático e utilizados em solo apresentam uma significante melhora na manutenção do equilíbrio. ${ }^{12,24}$

Estudos sobre esse tema ainda são bastante escassos, pois a maioria dos estudos comparam idosos sedentários versus idosos que praticam treinamento resistido ou treinamento resistido versus hidroginástica. Até o presente momento não foram encontrados estudos que mostram a comparação dos efeitos da fisioterapia aquática versus musculação terapêutica no equilíbrio e qualidade de vida de idosos, assim como o termo "musculação terapêutica", o qual ainda é pouco conhecido e mencionado na literatura. Em virtude disso, o presente estudo foi delineado com o intuito de contribuir com mais informações sobre este tema na tentativa de preencher essa lacuna de conhecimento.

\section{CONCLUSÃO}

Conclui-se que o grupo de fisioterapia aquática e musculação terapêutica apresentaram um melhor equilíbrio na escala de Tinetti quando comparado com os outros grupos. Em relação ao SF-36, a musculação terapêutica apresentou melhores resultados no estado geral de saúde e nos aspectos sociais e uma menor limitação por aspectos físicos. No entanto, o grupo 
fisioterapia aquática apresentou uma melhor saúde mental, aspectos emocionais e uma menor dor e a musculação terapêutica apresentou uma melhor vitalidade e uma melhor capacidade funcional, demonstrando que a atividade física, independentemente do tipo, promove efeitos benéficos na qualidade de vida.

\section{REFERÊNCIAS}

1 - Celich KLS, Creutzberg M, Gomes I. Envelhecimento com qualidade de vida: a percepção de idosos participantes de grupos de terceira idade. Revista Mineira de Enfermagem. 201014 (2).

2 - Dias CM, Florence RT, Lemos A. Ações de enfermagem na atenção primária e qualidade de vida do idoso: revisão integrativa. Revista de Pesquisa Cuidado é Fundamental. 2014, 6(4): 1630-1641 .

3 - Ministério da Saúde (BR). Portaria no 2.528, de 19 de outubro de 2006. Aprova a política nacional de saúde da pessoa idosa. Diário Oficial da União, Brasília (DF) 20 out 2006.

4 - Abreu, A. Fisioterapia geriátrica. Rio de janeiro: Shape, 2005.

5 - Gillespie LD. et al. Interventions for preventing falls in older people living in the community. Cochrane database of systematic reviews, 2012. 12(9): 7146.

6 - Gasparotto LPR, Falsarella GR, Coimbra AMV. As quedas no cenário da velhice: conceitos básicos e atualidades da pesquisa em saúde. Revista Brasileira de Geriatria e Gerontologia, 2014, 17(1): 201-209.

7 - Ruoti RG, Morris DM, Cole AJ. Reabilitação aquática. São Paulo: Manole;2000.

8- MANNA, Luana et al. Investigação do equilíbrio corporal em idosos. Revista Brasileira de Geriatria e Gerontologia, 2008, 11(2): 155-165.

9 - Nascimento LCG, Patrizzi LJ, Oliveira CCES. Efeito de quatro semanas de treinamento proprioceptivo no equilíbrio postural de idosos. Fisioter. Mov. Curitiba, 2012, 25(2):325-331.

10 - Da Cunha MF et al. A influência da fisioterapia na prevenção de quedas em idosos na comunidade: estudo comparativo. Motriz. Revista de Educação Física. UNESP, 2009. 15(3):527-536.

11 - Meireles AE et al. Alterações neurológicas fisiológicas ao envelhecimento afetam o sistema mantenedor do equilíbrio. Rev Neurocienc, 2010, 18(1):103-108.

12 - Coehn M, Parreira P, Baratella T. Fisioterapia aquática. São Paulo: Manole; 2011.

13 - Allendorf DB, Schopf PP, Gonçalves BC, Closs VE, Gottlieh GV. Idosos praticantes de treinamento resistido apresentam melhor mobilidade do que idosos fisicamente ativos não praticantes. Revista Brasileira de
Ciência e Movimento, 2014 24(1).

14 - Resende SM, Rassi CM, Viana FP. Efeitos da hidroterapia na recuperação do equilíbrio e prevenção de quedas em idosas. Rev Bras Fisioter.2008;12(1):57-63. 15 - AVELAR, Núbia CP et al. Efetividade do treinamento de resistência à fadiga dos músculos dos membros inferiores dentro e fora d'água no equilíbrio estático e dinâmico de idosos. Revista Brasileira de Fisioterapia, 2010, 14(3).

16 - Rissiani PSR; Leal RM, Vendrusculo AP. Efeito da hidrocinesioterapia na força muscular e na flexibilidade em idosas sedentárias. Fisioterapia em Movimento, 2017, 23(4).

17 - Silva ASD, Lima AP. Os benefícios da reabilitação aquática para grupos especiais. EFDeportes. com. Buenos Aires, 2011.

18 - Câmara LC, Bastos CC, Volpe EFT. Exercício resistido em idosos frágeis: uma revisão da literatura. Fisioterapia em Movimento, 2012, 25(2)

19 - Allendorf DB et al. Idosos praticantes de treinamento resistido apresentam melhor mobilidade do que idosos fisicamente ativos não praticantes. Revista Brasileira de Ciência e Movimento, 2015, 24(1):134-144. 20 - Medeiros RF, Costa VM, Chiapeta AV. Aplicação de musculação terapêutica no ganho de força muscular e amplitude de movimento de mmss em paciente com discopatia degenerativa submetida à técnica de artrodese cervical: estudo de caso. EfDeportes, Buenos Aires, 2011, 16(16).

21 - Assumpção, Claudio de Oliveira et al. Treinamento resistido frente ao envelhecimento: uma alternativa viável e eficaz. 2008.

22 - LUCAS W.C. Ricardo. Musculação Terapêutica. Santa Catarina: Sistema W allace Consultoria Ltda; 2010. 23 - André H, Ramalho F. Aptidão física e ocorrência de quedas em idosos praticantes de exercícios físicos. O envelhecimento populacional um fenômeno, 2017, p. 113.

24 - Souza L et al. Comparação dos níveis de força e equilíbrio entre idosos praticantes de musculação e de hidroginástica. Revista Brasileira de Atividade Física \& Saúde, 2014, 19(5):647-647.

25 - Civinski C, Montibeller A, Braz ALO. A importância do exercício físico no envelhecimento. Rev da Unifebe. 2011;9:163-75.

OBSERVAÇÃO: Os autores declaram não existir conflitos de interesse de qualquer natureza. 